\title{
Iter: de l'imaginaire du voyage à sa restitution visuelle et sonore
}

\author{
AURÉLIE HERBET
}

\begin{abstract}
Aurélie Herbet, doutoranda em artes plásticas e ciências da arte, Paris-1 - Pantheon-Sorbonne. Nasceu na cidade francesa de Neuville aux Bois, vive e trabalha em Paris. Mestre em Artes Plásticas pela Universidade de Paris-1 - Pantheon-Sorbonne em 2009. Atualmente cursa doutorado na mesma universidade sob a orientação do artista e professor doutor Bernard Guelton. Desenvolve pesquisa intitulada "Ficção e Criação Virtual: Territórios de Hipernarração". Seu trabalho de pesquisa, de caráter teórico-prático, se concentra sobre a ficção e suas modalidades narrativas e imersivas quando inseridas em espaços hipermidiáticos. Herbet também produz e experimenta dispositivos híbridos de espaços reais e virtuais. A artista atua em duas linhas de pesquisa ("Arte e Fluxo", dirigida por Yann Toma e "Ficções \& Interações" dirigida por Bernard Guelton) junto ao CERAP ( Centre d'Etude et Recherche em Arts Plastiques, Paris-1) no qual desenvolve seus temas de investigação. Associada desde 2011 ao programa de pesquisa EN-ER (Espace numerique en extension du réel) do ENSAD, Aurélie Herbert reflete sobre as questões da conservação e apresentação de obras de arte realizadas e inseridas em universos virtuais. E-mail: aurelie.herbet@yahoo.fr
\end{abstract}




\section{- RÉSUMÉ}

Cet article porte sur ma installation, Iter, réalisée dans le cadre de l'exposition Espaços outros. Menant une réflexion théorique et pratique sur la notion de fiction lorsque celle-ci est déplacée dans des espaces hypermédiatiques, je développerai ici trois axes de recherche qui animent Iter et plus largement, ma thèse. Ainsi, Iter est un dispositif faisant figurer un réseau évolutif généré par le récit de mes déplacements géographiques. A ce dispositif visuel s'ajoute un dispositif sonore, réalisé à partir de captations sonores prélevées dans les espaces rencontrés. Dans un premier temps il s'agira de s'interroger sur l'importance de la marche, du déplacement dans l'espace, pensés comme geste et performance artistique. Dans un second temps, je m'intéresserai à cet « effet de présence ", notion largement employée lorsque l'on évoque des pratiques dans le champ de l'art numérique. Je reviendrai sur ce terme en le définissant précisément et en observant comment cette notion est effective dans cette installation. Enfin, j'analyserai les rapports entre texte et image au sein du dispositif Iter. Nombreuses sont les œuvres numériques réalisées à partir de la " data visualization ». II s'agira d'en donner quelques exemples, mais aussi d'explorer les spécificités de ces œuvres.

\section{- MOTS-CLÉS}

Récit, voyage, tag, espace sonore, cartographie.

\section{- RESUMO}

Este artigo apresenta os resultados da minha instalação, Iter, realizada para a exposição Espaços Outros. Trata-se de uma reflexão teórica e prática sobre a noção de ficção abordada no contexto dos espaços hipermédia. Serão desenvolvidas três trajetórias de reflexão que inspiram o projeto Iter e, no sentido mais amplo, a totalidade de minha pesquisa.

Iter é um dispositivo que visualiza uma rede evolutiva gerada pela narrativa dos meus deslocamentos geográficos. Este dispositivo conta também com uma parte sonora, composta de gravações feitas nos espaços percorridos durante os deslocamentos. Inicialmente, trata-se de refletir sobre a importância do andar, do deslocamento no espaço, concebidos como gestos performáticos. Em seguida, me interessa o efeito de presença, um conceito amplamente utilizado quando nos referimos a práticas no campo da arte computacional. Este conceito será definido e analisado especificamente no contexto de Iter. Finalmente, será examinada a relação entre texto e imagem no dispositivo Iter. Atualmente, muitas obras computacionais usam os recursos de "visualização de dados". Alguns exemplos serão abordados para examinar suas especificidades.

- PALAVRAS-CHAVE

Narrativa, viagem, tag, espaço sonoro, cartografia.

Etonnement et déception des voyages. Illusion d'avoir vaincu la distance, d'avoir effacé le temps.

Etre loin.

George Perec, Espèces d'espaces, Paris, Galilée, 1974. p. 153.

\section{Introduction}

Depuis plusieurs années, je mène une réflexion théorique et pratique sur les dispositifs artistiques hypermédiatiques convoquant la notion de fiction. Cette recherche me mène à me questionner sur la place du spectateur au sein de ces œuvres dites de 
« fiction ». La fiction vient du latin fingere, qui veut dire à la fois « modeler », «façonner dans son esprit », « imaginer » mais aussi donnant le verbe « feindre ». L'hypermédia se définit par un media (vidéo, radio, internet, etc.) dans lequel sont diffusées plusieurs types d'information (image, son, vidéo ou encore multimédia). Mes dispositifs hybrident ainsi différents media et convoquent différents espaces (espaces tangible, fictionnel ou encore numérique). Ma problématique centrale est de savoir comment produire de la fiction, de l'imaginaire et immerger le spectateur au sein de ces dispositifs hybrides.

Cet article portera ainsi sur ma dernière installation, Iter, réalisée dans le cadre de l'exposition Espaços Outros. Je développerai ici trois axes de recherche qui animent Iter et plus largement ma recherche plastique.

Ainsi, Iter est un dispositif interactif faisant figurer un réseau évolutif généré par les mots-clés issus de mes récits de voyage. A ce dispositif visuel cartographique s'ajoute un dispositif sonore, réalisé à partir de captations sonores prélevées tout au long de mes déplacements. Dans un premier temps, il s'agira de convoquer l'imaginaire du voyage, le territoire inconnu comme un vecteur d'expérimentations plastiques et créateur de récits. Dans un second temps, je m'intéresserai à la marche et au déplacement dans l'espace, pensés comme geste et performance artistique. Dès lors, qu'implique la transposition d'un espace au sein d'un autre ? Quelles relations s'établissent entre le spectateur et le dispositif lorsque ce dernier est le résultat d'une «présence/absence » en constante évolution ? Cette dernière question sera l'occasion de convoquer la notion « d'effet de présence », très largement employée lorsque l'on évoque des pratiques dans le champ de l'art numérique. Je reviendrai sur ce terme en le définissant précisément et en observant comment cette notion est effective dans cette installation. Enfin, j'analyserai les rapports entre texte et image au sein du dispositif Iter. Nombreuses sont actuellement les œuvres numériques réalisées avec le procédé de la « data visualization ». II s'agira d'en donner quelques exemples, mais aussi d'explorer les spécificités de ces œuvres.

\section{L’imaginaire du voyage : espace autre et/ou hétérotopie?}

Pour l'exposition Espaços Outros j'ai choisi d'écrire et de représenter visuellement mes « récits de voyage » d'un territoire que je ne connais pas. Les voyages sont des moments privilégiés de découverte, d'inconnu, de questionnements et de nouvelles sensations. Un territoire inconnu est pour moi un « espace autre », un espace dans lequel je ne me « situe » pas, en d'autres termes dans lequel je n'ai plus les mêmes repères. Dès lors, cet espace, voire même ces espaces, donnent lieu à la création de fantasmes, d'imaginaire mais aussi d'inquiétude.

Espaces autres est également le titre d'une conférence donnée par Michel Foucault en 1966. Foucault qualifie ces espaces autres d'hétérotopies. Selon lui, les hétérotopies sont des espaces nés « dans la tête des hommes, dans l'interstice de leurs mots, dans l'épaisseur de leurs récits ou encore dans le lieu sans lieu de leurs rêves "'. Les hétérotopies sont des lieux, des utopies que l'on peut situer sur la carte, l'auteur les définit alors comme des localisations physiques de l'utopie, en d'autres

FOUCAULT, M. Le corps utopique, Les hétérotopies. Paris, Nouvelles Editions Lignes, 2009, p. 23. «Le corps utopiques » et «Les hétérotopies » sont des conférences données par Michel Foucault les 7 et 21 décembre 1966 sur la radio France-Culture. 
termes, des utopies situées : «Je crois qu'il y a - et ceci dans toute société - des utopies qui ont un temps déterminé, un temps que l'on peut fixer et mesurer selon un calendrier de tous les jours. II est bien probable que chaque groupe humain, quel qu'il soit, découpe dans l'espace qu'il occupe, où il vit réellement, où il travaille, des lieux utopiques, et, dans le temps où il s'affaire, des moments uchroniques "². Ces localisations se situent en-dehors de l'espace bien qu'elles soient situées : elles sont propices à l'imaginaire, à la découverte d'un ailleurs.

Cette définition d'hétérotopie, développée par Foucault se concentre sur des endroits bien précis : une cabane d'enfant, un cimetière, un centre pénitencier, etc. En somme, dans des lieux ayant une fonction précise. Je distinguerai dès lors ici les notions d'espace autre et d'hétérotopie, pour ne conserver que la première, la seconde étant selon moi hors de propos quant à mon travail plastique. J'entendrai ici par Espace autre, un espace dans lequel l'altérité d'un nouvel espace est présente, un espace qui m'est étranger, c'est-à-dire en dehors de ma conception commune.

Avant la présentation d'/ter, il est important de préciser que je ne considère pas que tous voyages, que tout espace inconnu, sont des espaces autres. Ma démarche artistique m'a conduit à considérer ce voyage comme tel en établissant un processus et en portant un autre regard sur ce que peut être la découverte d'un territoire, en cherchant dans chacun des lieux que je découvrais ce qui pouvait abriter de l'imaginaire, susciter un interstice entre l'espace perceptif et l'espace imaginaire et en se focalisant sur l'altérité d'un espace méconnu.

Iter, qui signifie en latin chemin, trajet, voyage, est une production artistique qui se scinde en deux parties très distinctes. Mon travail plastique se situe ainsi à la fois sur le terrain, là où je me trouve géographiquement, et dans l'espace de la galerie. Ces espaces autres sont la matière à la construction d'un réseau de mots évoluant au fil de mes trajets. En premier lieu, ma démarche s'est concentrée sur le terrain et a consisté à écrire, chaque jour, les récits de ce que je percevais autour de moi. Pendant une dizaine de jours, durée de mon voyage, j'ai donc observé et noté mes sensations olfactives, auditives, gustatives, tactiles et visuelles. Ces récits décrivent ma vision, ma lecture subjective de ce nouveau territoire, que j'appréhendais et découvrais au fur et à mesure de mes déplacements. Dans un même temps, je capturais, à l'aide d'un enregistreur, les bruits, les sons, les conversations, la musique qui se déployaient dans ces espaces. Ces traces sonores et écrites font l'objet d'une installation combinant à la fois une projection et une bande-son, actualisée chaque jour.

Sur la projection figurent ainsi des « mots-clés » matérialisés par des points. Ces points ne sont pas placés au hasard : la carte se décompose en 5 zones, correspondant aux 5 sens énoncés précédemment. Chaque mot, relatif à une sensation corporelle, est donc placé suivant le sens convoqué : se dessine alors un réseau déterminé par les aires sensorielles. Par ailleurs, le diamètre de ces points augmente en fonction du nombre d'entrées d'un même mot : plus le mot sera convoqué, plus le « tag » sera important. Au fur et à mesure de l'écriture de ces récits, les mots sont enregistrés et répertoriés dans une base de données me servant à les classer suivant leur champ lexical. Plus un mot se rapproche du champ lexical d'un autre plus il est proche et inversement.

Lors du début de la mise en œuvre de mon projet, je me suis inspirée de la méthode de l'atlas sémantique existant sur le site internet dico.cnrs. Cet atlas séman- 
tique propose pour « chaque entrée du dictionnaire des synonymes, des liens analogiques et un modèle pour la représentation géométrique des valeurs sémantiques des différents termes ». II s'agit avec cet atlas de visualiser la distance sémantique entre plusieurs mots. Ces mots sont en relation les uns aux autres et forment ainsi des ensembles de sens. Prenant au départ comme référence ce modèle, j'ai fait le choix ensuite de m'en éloigner afin d'établir ma propre carte issue de mes récits de voyage mais aussi de mes sensations ressenties pendant ce déplacement. La cartographie générée ne résulte donc plus d'une visualisation des champs sémantiques mais d'associations entre les différents sens.

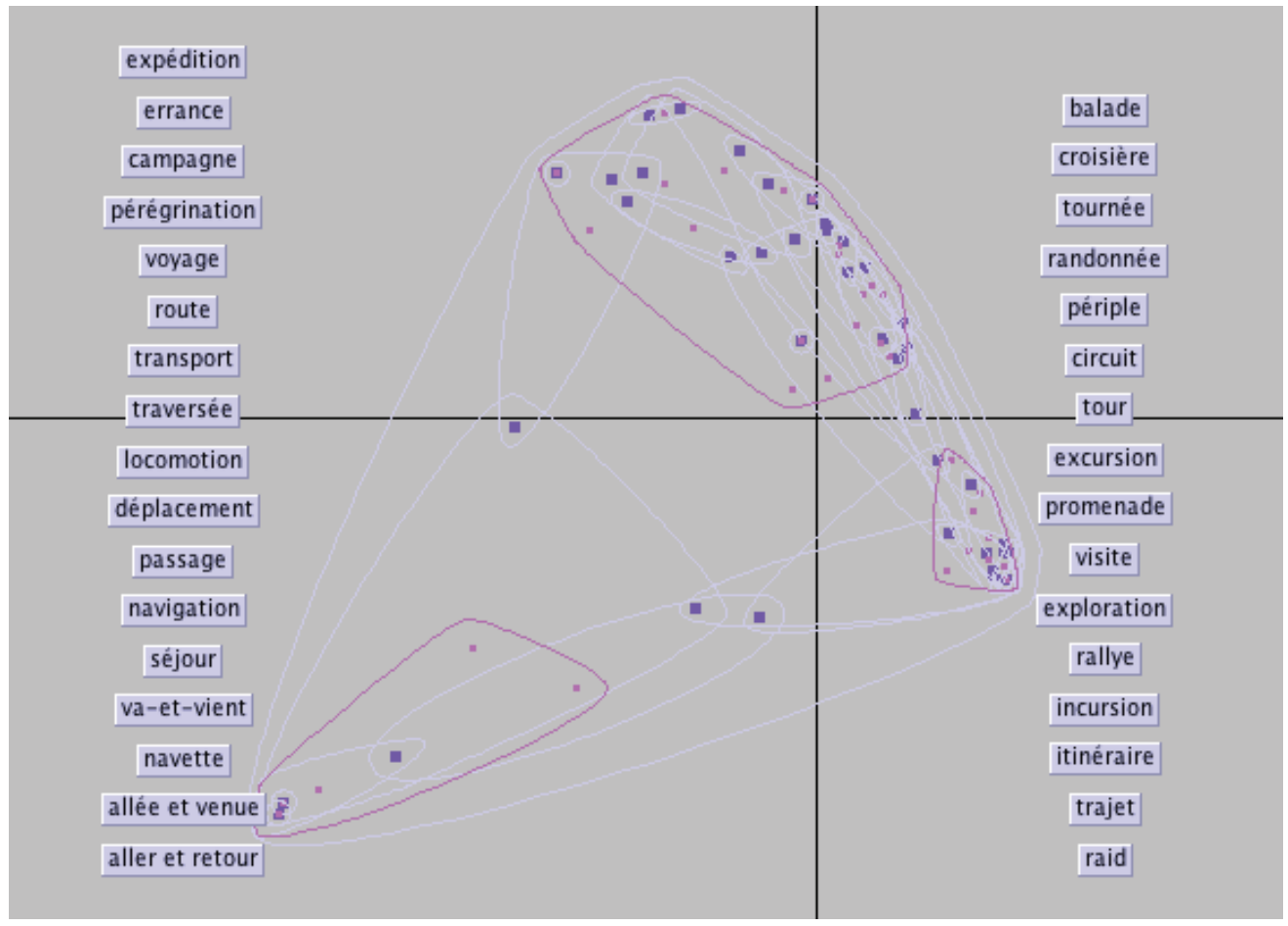

Figure 1- Capture d'écran du site http://dico.isc.cnrs.fr/fr/index.html. Atlas sémantique sur le thème du voyage.

Cette première partie a été l'occasion de définir et de bien déterminer ce que j'entendais par espace autre dans le cadre de mon travail. II a été nécessaire de considérer qu'ici un espace autre n'est pas à entendre en tant qu'hétérotopie, telle que l'a pensé Michel Foucault. II s'agira à présent de s'interroger sur l'importance de la marche, du déplacement dans l'espace, conçus comme geste et performance artistique. Plusieurs problématiques sont en jeu dans ce travail : comment restituer la sensation de différents espaces appréhendés au sein d'un autre espace ? Comment rendre compte d'un déplacement au sein d'une installation artistique ? Les traces sonores collectées ont-elles une valeur de document, de fragment de l'œuvre ou font-elles sens individuellement? 


\section{Le déplacement, la marche comme matériau artistique}

La marche et mes déplacements sont ainsi les matériaux de mon installation. Ils me fournissent de la matière à l'élaboration de mon dispositif plastique. La marche est une action. Le corps, par la marche appréhende l'espace qui l'environne. Maurice Merleau-Ponty note dans sa Phénoménologie de la perception combien la vision est relative au mouvement. Le corps est partie prenante du monde qui l'entoure. Alain Berthoz, professeur en Physiologie de la perception ajoute que « L'espace n'est pas un concept extérieur au cerveau de l'homme, il est perçu et il est vécu».3 ${ }^{3}$ Notre relation à l'espace passe alors par le corps en tant qu'il est le récepteur de cet espace, il passe alors par une connaissance, une analyse perceptive de l'espace qu'il y a autour de nous. Cette analyse conduit le corps à se faire sa propre perception, c'est ce que l'on appelle l'espace perceptif. Nous distinguons alors une spatialité de position, celle qui nous localise objectivement et une spatialité de situation, celle qui nous spatialise subjectivement. Le déplacement du corps, la marche est le moteur de cette spatialité de situation. Elle nous permet de nous inscrire dans notre espace et de le percevoir différemment. «Mouvoir son corps, c'est viser à travers lui, les choses. Notre perception reconstruit un espace invisible ". ${ }^{4}$ L'expérience de l'espace par le corps en mouvement constitue ainsi notre perception de notre propre corps. Par le déplacement, l'Homme se constitue et apprécie son espace corporel mais aussi l'espace qui l'entoure. La marche a donc une importance essentielle à l'entreprise de la connaissance du corps mais aussi à l'appréciation de ce qu'il y a autour. Par la marche, le corps crée son espace, circonscrit un territoire. Nombreux sont les artistes à avoir questionner cela.

Ainsi, comme l'explique Thierry Davila dans son ouvrage Marcher, Créer, Déplacements, flâneries, dérives dans l'art de la fin du XXe siècle, le déplacement dans l'espace a très souvent été une source de création pour les artistes et ce, depuis la Renaissance. Marcher est ainsi une action qui devient le médium de la création d'une œuvre:

Car tel est, dans le domaine de l'art, le destin de la déambulation : elle est capable de produire une attitude ou une forme, de conduire à une réalisation plastique à partir du mouvement qu'elle incarne, et cela en dehors et complément de la pure et simple représentation de la marche (iconographie du déplacement), ou bien elle est tout simplement elle-même l'attitude, la forme ${ }^{5}$.

Le déplacement est ici le producteur d'œuvres artistiques. En effet, la marche, depuis le $19^{e}$ siècle, a été un sujet d'expériences artistiques variées. On peut ainsi citer les flâneries de Baudelaire, les errances des surréalistes ou encore les dérives des situationnistes donnant lieu notamment à leur carte de psychogéographie résultant de leur « laisser aller aux sollicitations du terrain et des rencontres qui y correspondent ». Plus récemment, depuis les années 1970, Hamish fulton, artiste conceptuel rattaché au Land art et qualifié « d'inventeur de l'art de la marche », parcourt à pied le monde. Durant ses voyages, il ne collecte rien, aucun objet. Seuls ses photographies et ses récits, ses descriptions d'itinéraires, retracent son expérience. Le spectateur

\footnotetext{
BERTHOZ, A. Le sens du mouvement. Paris, éditions Odile Jacob Sciences, 1997.

4 Ibid., p. 304.

DAVILA, T. Marcher, Créer, Déplacements, flâneries, dérives dans l'art de la fin du XXe siècle. Paris,

Editions du Regard, 2002, p. 15.
} 
est invité à reconstituer l'espace évoqué par le parcours de l'artiste. Ces artistes, et en particulier Fulton, questionnent le corps et son implication au sein de l'espace naturel ou urbain. Avec ces pratiques, ils tentent de comprendre comment l'être humain s'inscrit dans son espace et comment il interagit avec lui.

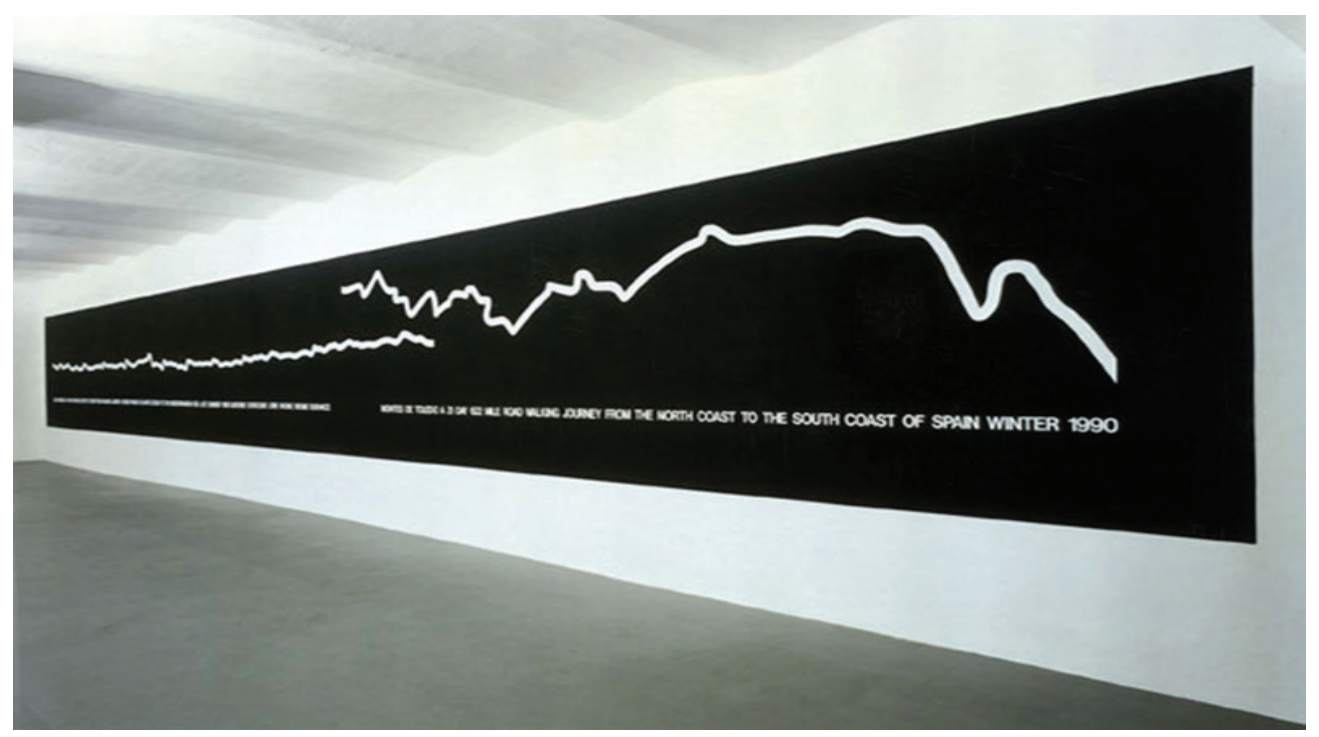

Figure 2 - Hamish Fulton, Horizon to horizon, 1990.

Pour continuer notre étude sur le mouvement du corps considéré comme médium il est important de définir ce que nous entendons par la notion de lieu et de bien le circonscrire au regard des pratiques artistiques étudiées. Pour cela, nous nous appuierons sur Écoumène : introduction à l'étude des milieux humains, d'Augustin Berque, géographe français. Berque étudie la distinction entre les deux étymologies grecques du lieu, le topos et la chôra. Ainsi, le topos désigne le lieu physique, cartographiable tandis que la chôra désigne le lieu existentiel. Cette dernière définit « un lieu dynamique, à partir de quoi il advient quelque chose de différent, non pas un lieu qui enferme la chose dans l'identité de son être ${ }^{6}$ ». Topos et chôra se lient continuellement. Pour Iter, ces deux définitions sont à prendre en compte : les endroits découverts sont mes terrains d'action, mes lieux d'existence au sens où Augustin Berque les définit. Le lieu se pose donc en dialectique en tant qu'il se situe entre espace réel, social et qu'il dévie vers un autre espace, celui-ci imaginaire. Iter, engage également la réflexion du corps, de son implication physique au sein d'un espace que cela soit par rapport à moi ou au spectateur face à l'installation. Par le mouvement du corps arpentant les lieux, nous nous mesurons à eux et nous les considérons : « Marcher devient le moyen privilégié pour écouter le monde, y prêter attention, parce que se déplacer est aussi une façon de se mettre à entendre ${ }^{7}$ ».

Afin de conclure sur cette question, il me semble souhaitable de convoquer brièvement la notion de cinéplastique, inventée par Elie Faure en 1920. Thierry Davila reprend cette notion, initialement utilisée pour décrire le cinéma : 
Il faut considérer la cinéplastique comme la tentative de ne pas réduire le mouvement à la translation, à la pure et simple mobilité physique, mais bien au contraire comme la prise en charge du mouvement, des déplacements, dans toute leur ampleur, dans toutes leurs manières, y compris dans leurs dimensions psychiques et fantasmatiques ${ }^{8}$.

Le mouvement, la marche sont la matière créative pour de nombreux artistes ; mais inversement, leurs dispositifs plastiques engendrent et produisent des mises en forme du lieu dans un contexte déterminé. Iter, par mes déplacements, tente de produire une réflexion sur le lieu dans lequel le visiteur se trouve. II ne s'agit pas simplement de montrer des traces, de produire une documentation d'un voyage, de restituer des sensations dans l'espace d'exposition mais de symboliser un trajet et des rencontres.

\section{Iter: du récit à sa visualisation spatiale}

De mon arrivée au Brésil jusqu'à mon départ, j'ai ainsi écrit, chaque jour, les récits de mes déplacements : dans ces récits sont décrits mes ressentis et mes sensations par rapport aux espaces rencontrés. Chaque jour de nouveaux mots émergent et viennent créer, au sein de la galerie, un réseau renouvelé. Les données utilisées ont été préalablement sélectionnées et collectées dans une base de données réalisée au fur et à mesure de mes déplacements. Un chemin, un réseau, s'établit ainsi entre tous ces mots relatifs aux différents champs lexicaux en relation à ce que je vois et ressens. Ces mots ne sont plus lisibles au sein de l'installation. Seule leur spatialisation liée à leurs rapports sensoriels et leurs interconnexions, sont visibles. Cette installation convoque à la fois un ici, l'espace de la galerie dans lequel est projetée l'installation et des ailleurs, les différents lieux rencontrés. Les captations sonores diffusées dans la galerie et prélevées quotidiennement, peuvent donner des indices des différents lieux traversés sans pour autant dévoiler les récits. Un mystère entoure alors l'œuvre : le spectateur est invité à imaginer les lieux et les situations que j'ai rencontrés ; ou à se construire ses propres récits de voyage. L'installation immerge ainsi dans un dispositif de type englobant : le spectateur peut à la fois écouter et percevoir ce qui se trame dans cette installation. Les sons lui permettent d'être à la fois présent au sein de la galerie mais le projette également vers d'autres espaces qu'il ne peut pas appréhender de manière directe : il les perçoit à travers son écoute et son imagination. En explorant à la fois ce territoire sonore et cette cartographie abstraite, les visiteurs se situent dans un entre-deux. Entre le texte sous-jacent, mes récits, et leurs représentations visuelles et auditives (la cartographie et les fragments sonores). Ainsi, comment s'effectue, dans ce dispositif, le passage du texte à l'image ?

La « data visualization », la visualisation de données est l'étude de la représentation visuelle des données. Elle est actuellement de plus en plus employée dans le but de communiquer clairement et rapidement de l'information à l'aide de schémas visuels. Lev Manovich dans The langage of new media, affirme que les bases de données sont une forme dominante des nouveaux medias. Avec l'essor de la data visualization, de plus en plus d'artistes s'emparent de ces données et les utilisent comme sujet ou medium pour leur recherche artistique. Parmi ces artistes, on peut citer Benjamin Fry qui,

8 Ibid., p. 27. 
en 1999, réalise l'œuvre Valence. Cette œuvre met en évidence graphiquement les liens qu'entretiennent certains fragments d'informations et la manière dont ils interagissent ensemble. Valence est un logiciel effectuant la lecture d'un texte choisi et représente tridimensionnellement la relation qu'entretiennent les mots par l'intermédiaire de segments et la distance qui les séparent. Ainsi, l'image de cette page est tirée d'une visualisation du contenu du livre The innocent abroad de Mark Twain. Le programme lit le livre d'une façon linéaire, ajoutant dynamiquement chaque mot sur la carte.

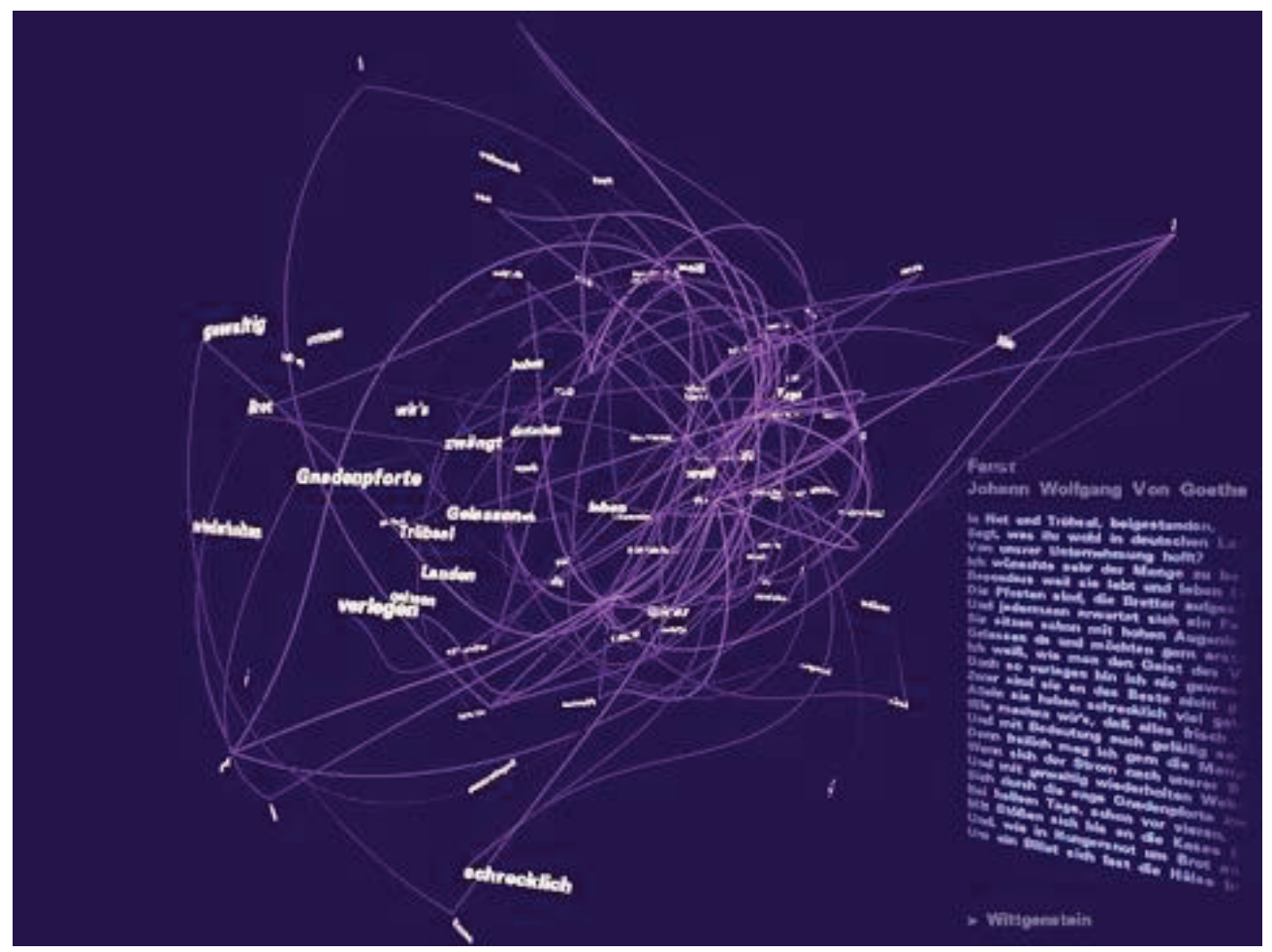

Figure 3 - Benjamin Fry, Valence ${ }^{9}$, 2002. (c) Copyright Ben Fry and the Massachusetts Institute of Technology.

Pour Iter cette visualisation de données est poétique et non informative. Pour cette constellation issue d'une base de données en constante évolution, j'ai fait le choix de ne pas faire figurer les mots. Le spectateur ne peut ainsi pas s'informer sur les récits que j'ai produits. Seuls les tags (les ronds noirs) sont les indices de ces mots mais ne les dévoilent pas. Ils proposent une vision subjective d'autres espaces, la cartographie ne me localise pas et ne divulgue pas son contenu. Cette cartographie est fictive, dans le sens où elle véhicule de la fiction, des histoires, mais qui ne sont plus visibles. La constellation se génère au fur et à mesure des jours et forme une carte abstraite, une carte sur laquelle aucune donnée tangible n'est montrée. Se crée une carte paysagère au sens où la définit Anne Cauquelin, théoricienne de l'art dans son ouvrage Le site et le paysage. Une carte paysagère n'est pas une carte topologique, statique, répertoriant différents points d'un territoire. Une carte paysagère

9 Consulté sur http://benfry.com/valence/ le 6/04/2012. 
est établie ou dressée par rapport à un point de vue. C'est une carte en constante mutation : «il ne s'agit donc pas de cartographier des réseaux fixes, mais des actions

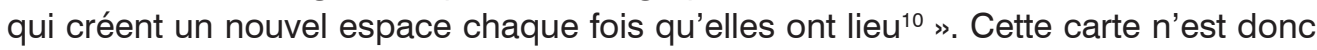
pas calculable métriquement mais se dessine par les différentes actions. Avec ce type de carte l'on ne cherche plus à cartographier statiquement mais dynamiquement. La carte fait ainsi figurer le corps en mouvement, ses déplacements.

\section{Conclusion}

Pour l'exposition Espaços Outros j'ai souhaité proposer une vision cartographique et fragmentée du voyage. Voyage mental, voyage dans des lieux existants ou imaginaires. "L'espace sous-entend à une forme d'écriture et de lecture du monde ${ }^{11}$ » explique Paolo Amadi dans son livre Espaces analysant la question de l'espace à travers les siècles. Avec cette installation j'ai voulu faire partager ma découverte d'un territoire qui m'est complètement inconnu, étranger : l'étranger, qui provient du latin extraneus, signifie " ce qui est à l'extérieur » : cette découverte d'un nouveau territoire fut pour moi l'occasion d'expérimenter et d'appréhender ces espaces d'une autre manière. Par ces récits j'ai tenté de retranscrire ce qui m'entourait. Le carnet de voyage contenant ces récits n'est pas visible au sein de l'installation. Deux éléments indiciels étaient présents : d'une part, la cartographie processuelle, suscitant alors curiosité et imaginaire de la part du visiteur, qui se construit à son tour une cartographie imaginaire des espaces l'environnant. D'autre part, la bande-son, résultat de ces captations sonores, de ces fragments collectés lors de mon voyage ; elle accompagnait la constellation de mots-clés et invitait le spectateur à percevoir par le son des fragments d'autres espaces, des fragments du monde :

Le monde, non plus comme un parcours sans cesse à refaire, non pas comme une course sans fin, un défi sans cesse à relever, non pas comme le seul prétexte d'une accumulation désespérante, ni comme une illusion d'une conquête, mais comme retrouvaille d'un sens, perception d'une écriture terrestre, d'une géographie dont nous avons oublié que nous sommes les auteurs ${ }^{12}$.

\section{Références}

AMALDI, Paolo. Espaces. Paris, Editions de la Villette, 2007.

BAUDELAIRE, Charles. Curiosités esthétiques. L’art romantique. Paris, Garnier, 1986.

BENJAMIN, Walter. Paris, capitale du XIXe siècle : Le livre des passages [1982], trad. J.Lacoste, Paris, Payot, 1989.

BERQUE, Augustin. Écoumène : introduction à l'étude des milieux humains. Paris, Belin, 2000.

\footnotetext{
${ }_{10}$ CAUQUELIN, A. Le site et le paysage. Paris, éditions PUF, 2002, p. 99.

11 AMALDI, P. Espaces, Paris, Editions de la Villette, 2007, p. 16.

12 PEREC, G. Op. cit., p. 156.
} 
BERTHOZ, Alain. Le sens du mouvement. Paris, éditions Odile Jacob Sciences, 1997.

CAUQUELIN, Anne. L'invention du paysage. Paris, Plon, 1989.

CAUQUELIN, Anne. Le site et le paysage. Paris, éditions PUF, 2002.

DAVILA, Thierry. Marcher, Créer, Déplacements, flâneries, dérives dans l'art de la fin du XXe siècle. Paris, Editions du Regard, 2002.

MANOVICH, Lev. Le langage des nouveaux médias. Paris, Les presses du réel, 2010.

MERLEAU-PONTY, Maurice. Phénoménologie de la perception. Paris, Gallimard, 1945.

PAQUOT, Thierry et YOUNES, Chris. Espace et lieu dans la pensée occidentale : de Platon à Nietzsche. Paris, éditions La découverte, 2012.

PEREC, Georges. Espèces d'espaces. Paris, Editions Galilée, 1974.

FAURE, Elie. De la cinéplastique [1920]. Paris, Séguier, 1995.

FOUCAULT, Michel. Le corps utopique, Les hétérotopies. Paris, Nouvelles Editions Lignes, 2009.

SCHELLE, Karl Gottlob. L'art de se promener [1802]. Paris, Payot \& Rivages, 1996.

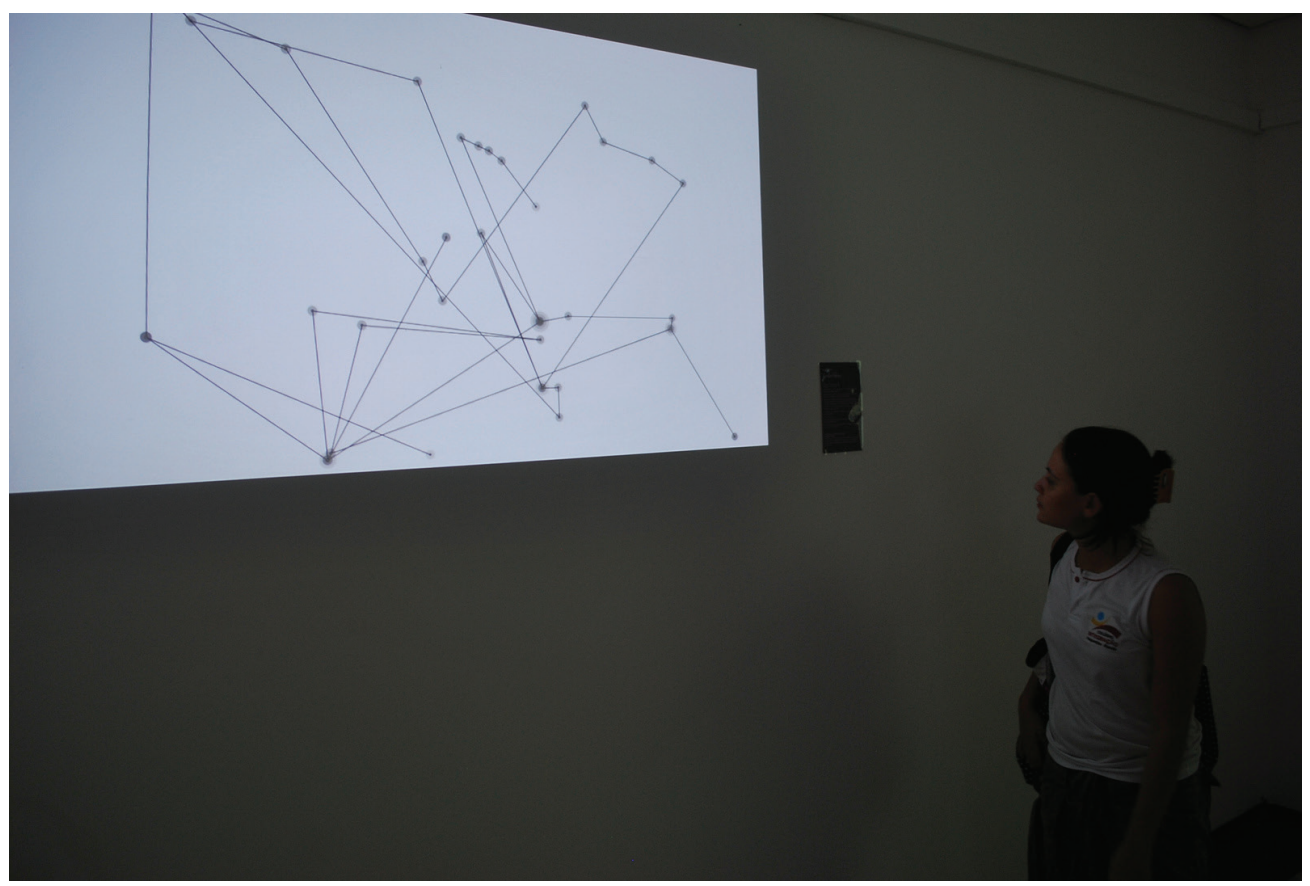




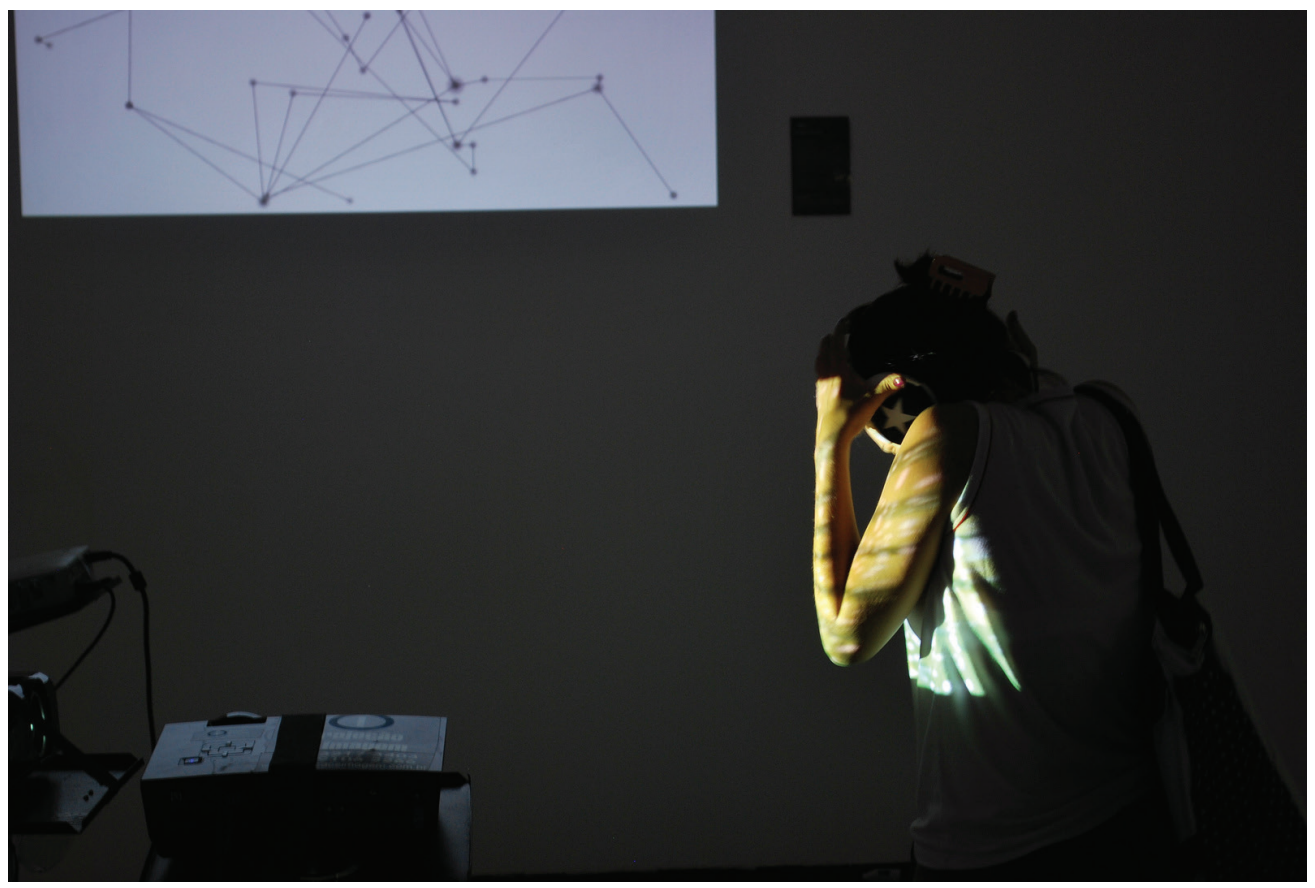

Figure 4 - Vues de l'installation Iter au sein de l'exposition Espaços Outros, Galeria de arte Ido Finotti, (C) Paulo Rogerio Luciano.

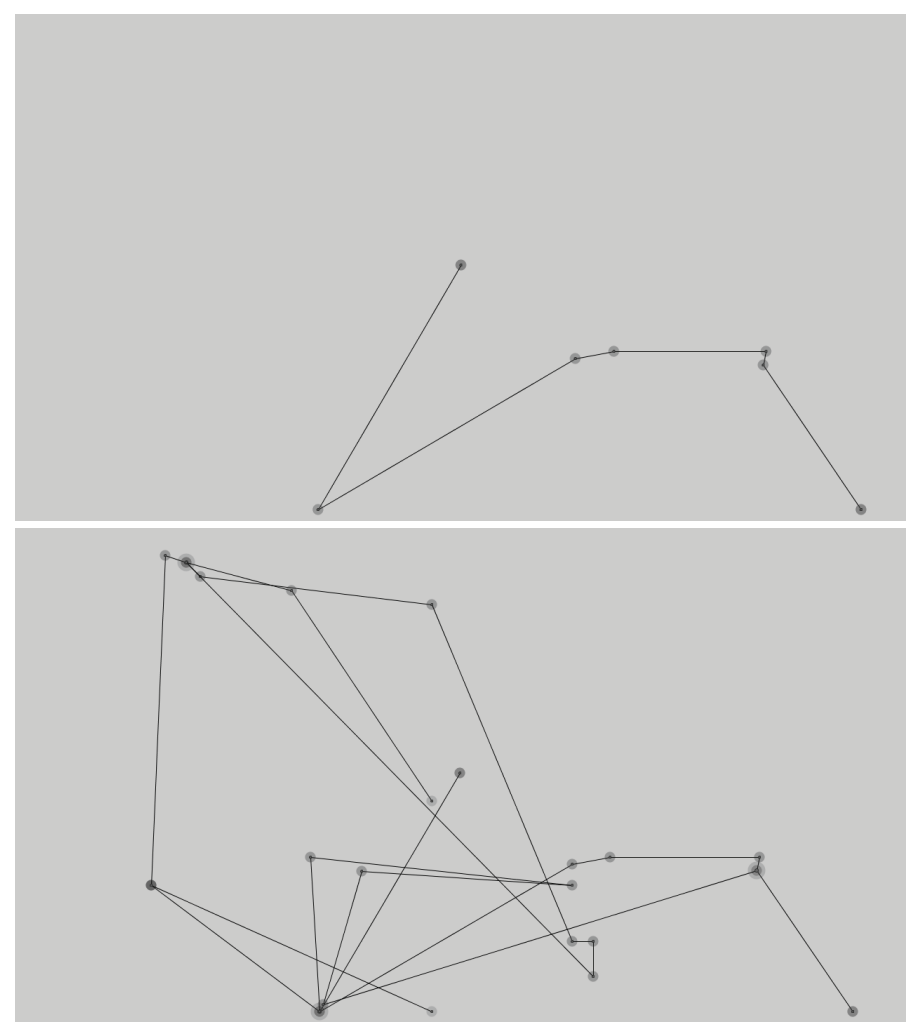



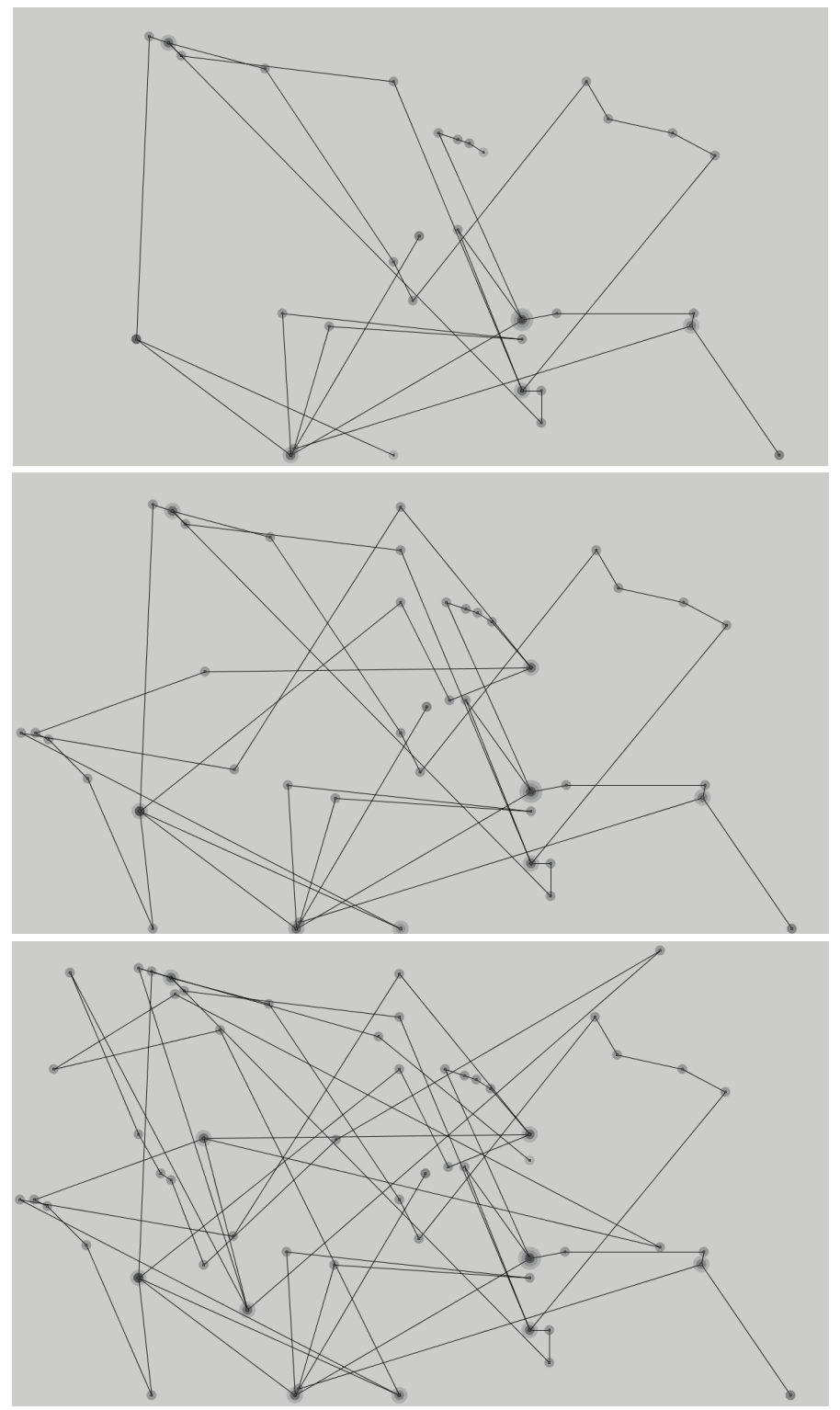

Figure 5 - Evolution du dispositif Iter au fil des jours, Aurélie Herbet, courtesy de l'artiste. 